\title{
Implicit Attitudes Toward Foreign Accents
}

\section{Joshua Daniels}

University of Florida

Faculty mentor: Edith Kaan, Department of Linguistics

\begin{abstract}
Over the past 20 years, the Implicit Attitude Test (IAT) has become a robust paradigm for evaluating unconscious biases about socially sensitive topics (implicit attitudes) in a way that avoids social desirability bias. It has been widely applied to the psychology surrounding race, gender, and other social characteristics, and is a potentially useful tool for linguists particularly in studying the effects of dialect and accent. This study demonstrates an application of the IAT to accents, particularly examining implicit attitudes about Chinese-accented English relative to Standard American English. The study evaluated participants' implicit attitudes about Chinese accents using the IAT, measured their explicit attitudes using the Speech Evaluation Instrument (SEI) developed by Zahn and Hopper (1985), and recorded selfreported rates of exposure to Chinese accents. It found that participants had both implicit and explicit attitudes toward Chinese-accented English that were significantly negative relative to American English. It did not, however, find a correlation between participants' explicit and implicit attitudes. There was a weak correlation between implicit attitudes and self-reported rates of exposure to Chinese accents, although the interpretation of this finding is unclear.
\end{abstract}

Keywords: implicit attitudes, implicit attitude test, foreign accent, Chinese accent

\section{Introduction}

The theoretical concept of implicit attitudes, also known as implicit biases or implicit associations, was first introduced in 1998 by Greenwald, McGhee and Schwartz. It was intended to extend previous work on implicit cognition to the topic of social biases and attitudes. In the same paper, Greenwald, McGhee and Schwartz introduced an experimental paradigm to evaluate individuals' implicit attitudes: the Implicit Attitude Test (IAT). This test takes the form of a combined sorting task in which participants are asked to sort two different categories of stimuli at the same time. As a simple example, participants might be presented with a combined set of 
both shapes and characters one at a time and asked to sort squares and letters into one category while sorting circles and numbers into another, or vice versa. In this example, if a participant has an implicit association between squares and letters, they should find it easier to sort them into the same category and more taxing to have to sort them into different categories. The difference in a participant's reaction time can be taken as a measure of the strength of their associations between the stimuli being tested. In practice, the IAT is generally used to evaluate attitudes about social categories and interpersonal characteristics rather than associations between shapes and characters.

The current study applied the Implicit Attitude Test to US-English speakers' attitudes about Chinese and American accents. The goals of the study were to determine if there are implicit biases against Chinese-accented English relative to Standard American English, to determine the extent to which there is a correlation between implicit and explicit attitudes toward Chineseaccented English, and to determine the extent to which implicit and explicit attitudes about Chinese accents correlate with previous exposure to such accents. We expected to find both implicit and explicit attitudes to be negative overall. We also expected that negative explicit attitudes would correlate with more negative implicit attitudes, and that more frequent accent exposure would correlate with less negative implicit attitudes.

\section{Literature Review}

Since being introduced by Greenwald, McGhee, and Schwartz (1998), use of the IAT has expanded greatly. It has become a staple of social psychology research, especially regarding the psychology of race (Haider et al., 2015), gender (Dunham, Baron, \& Banaji, 2016), religion (Pirutinsky, Carp, \& Rosmarin, 2017), and sexuality (Westgate, Riskind, \& Nosek, 2015). The IAT is particularly good for investigating socially sensitive topics because results have been shown to be resistant to intentional modification by participants (Egloff \& Schmukle, 2002). This frees it from many of the social desirability concerns of more explicit measures and allows it to tap potentially unconscious attitudes. The IAT is also useful because there is a large and continually growing body of research documenting many of its practical characteristics as a psychometric measure. These include both its reliability and validity (Greenwald, Nosek, \& Banaji, 2003), its sensitivity to order effects and the effects of differing numbers of stimuli (Nosek, Greenwald, \& Banaji, 2005), and the degree to which it is influenced by participant 
exposure to other psychometric measures (Greenwald, Poehlman, Uhlmann, \& Banaji, 2009). Significant effort has also been made to establish a more reliable, evidence-based scoring algorithm, including recommendations for proper treatment of errors and outliers (Greenwald, Nosek, \& Banaji, 2003).

These practical methodological foundations, along with the versatile and adaptable nature of the IAT, have contributed to its wide use in social psychology. Outside of social psychology, the IAT has begun to be applied to political science (Ksiazkiewicz \& Hedrick, 2013), political psychology (Friese, Smith, Plischke, Bluemke, \& Nosek, 2012), and even more recently has begun to find use in market research and product development (Gregg \& Klymowsky, 2013). While it is beginning to find traction in other fields, the IAT has seen little use in either psycholinguistics or sociolinguistics, despite their relative proximity to social psychology as a field. The IAT was used both by Pantos and Perkins (2012) as well as by Rosseel, Speelman, and Geeraerts (2018) to evaluate attitudes toward single accents relative to a standard dialect. Pantos and Perkins (2012) evaluated American participants' attitudes toward Korean-accented English relative to standard American English, while Rosseel, Speelman, \& Geeraerts (2018) evaluated Belgian attitudes toward regional varieties of Dutch relative to standard Dutch. The most notable novel linguistic application of the IAT comes from a sociolinguistic study that considered associations between various phonetic features and regions of the United States in an attempt to map out the salient aspects of various regional accents (Campbell-Kibler, 2012). Nevertheless, the overall linguistic application of the IAT has been restricted to disconnected projects like these. While social psychology has applied IAT measures to a broad range of topics, and political science is beginning to do the same, psycho- and sociolinguistics have not yet developed a similar body of research. In the present study, we try to contribute to the data base by applying the IAT to attitudes towards foreign accent speech.

\section{Methods}

\section{Main Study}

overview. The main study consisted of four tasks. First, participants completed Speech Evaluation Inventories (SEI; Zahn \& Hopper, 1985) for an American English speaker and a Chinese-accented speaker. Then, an IAT was conducted evaluating implicit attitudes toward Chinese accents as compared to an American English baseline. Participants then completed a 
modified Language Experience and Proficiency Questionnaire (LEAP-Q; Marian, Blumenfeld, \& Kaushanskaya, 2007), which evaluated their language backgrounds as well as their selfreported level of exposure to the Chinese accents. Finally, a short debriefing questionnaire was completed to explain the experiment's purpose and identify ways to improve participant experience in future.

participants. In total, 26 participants took part in this study. Participants were recruited from the University of Florida student population and were compensated with either course credit for an applicable course, or payment at a rate of $\$ 7.50$ an hour. The participants ranged in age from 18 to 28 , with a median age of 20 . Of the participants, 18 were women.

implicit association task (IAT). The accent version of the IAT using auditory stimuli in this study is modeled on the IAT paradigm used in Pantos (2012). The use of auditory stimuli in IATs is relatively uncommon, but it is essential when applying these tests to accent. The auditory stimuli used consisted of eight neutral three-syllable phrases. The visual stimuli used were eight positive valence words and eight negative valence words, all taken from those used by Pantos (2012). All stimuli are listed in Table 1 below:

Table 1. Stimuli for IAT

\begin{tabular}{|c|c|c|}
\hline \multicolumn{2}{|c|}{ Visual stimuli } & \multirow[t]{2}{*}{ Auditory stimuli } \\
\hline Positive valence & Negative valence & \\
\hline marvelous & tragic & around the world \\
\hline superb & horrible & show you that \\
\hline pleasure & agony & think through this \\
\hline beautiful & painful & different thing \\
\hline joyful & terrible & very large \\
\hline glorious & awful & join a club \\
\hline lovely & humiliate & yesterday \\
\hline wonderful & nasty & try to find \\
\hline
\end{tabular}

*Visual stimuli taken from those used by Pantos and Perkins (2012)

Each auditory stimulus was recorded separately twice: once by a speaker with an American English accent and once by a speaker with a Mandarin Chinese accent. Both speakers were female students at the University of Florida. The American English speaker was a 22-year-old 
native English speaker with a Standard American English accent. The Chinese-accented speaker was also 22 years old, originally from Zhengzhou in the Henan Province of China, and spoke Mandarin as her native language. She moved to the United States in 2016 and had begun learning English at age 15. In both cases, the audio stimuli were recorded in a sound-isolated booth using a Marantz PMD660 Digital Recorder with a sampling frequency of $44.1 \mathrm{kHz}$ and a 24-bit sampling rate. Speakers recorded each phrase three times in sequence and the most natural sounding sample was selected and isolated. The volume of all audio stimuli was then normalized.

A small norming survey was conducted with Qualtrics survey software in order to confirm the intelligibility of the auditory stimuli and to test the identifiability of the accents. Twenty participants were recruited through Amazon's Mechanical Turk. The survey excluded any selfreported non-native English speakers, as well as anyone attempting to complete it from a nonAmerican IP address. Before beginning, participants reported their age and gender: seven were female; ages ranged from 22 to 56; the median age was 33.

For the norming survey, participants were presented with the 16 accented audio stimuli and were first given free response fields and asked to identify both the content of the phrase and its accent. In a second portion, participants were presented with the same 16 auditory stimuli, but this time were only asked to identify the accent and were given a choice between American English and Mandarin Chinese-accented English.

The results of this study indicate both the content and accent of the stimuli were generally identifiable. Seventeen out of the 20 participants correctly identified what was said in every trial, and all correctly identified over $85 \%$ of the phrases. Participants were able to correctly identify the American accent in every trial, and most were able to identify the Chinese accent. While several participants misidentified the Chinese accented speech as Korean or Japanese, all participants correctly discriminated between the two accents when presented with fixed responses.

When audio stimuli were presented to participants in the main study, they were repeated three times with a second of silence inserted between repetitions, as was done in Pantos and Perkins (2012), although participants could respond from the beginning for every stimulus. A small sound indicator was presented on screen to indicate that an audio stimulus was being played. Visual stimuli remained on screen until the participant made a response. Upon an incorrect response, a red $\mathrm{X}$ was presented on the bottom of the screen which remained until participants 
corrected their response. Correct responses were followed by a 250-millisecond blank screen, then by a 250 millisecond fixation cross in the middle of the screen before the next trial began.

Each IAT consisted of seven blocks - B1 through B7 - as illustrated below in Table 2:

Table 2. Sequence of blocks for IAT

\begin{tabular}{llllr}
\hline Block & Left category (E) & Right category (I) & Stimuli presented & \# of trials \\
\hline B1 & Positive word & Negative word & Visual valence words & 32 \\
B2 & American accent & Chinese accent & Auditory accented phrases & 32 \\
B3 & Positive word or American & Negative word or Chinese & Visual valence words and & 64 \\
& accent & accent & auditory accented phrases & \\
B4 & Positive word or American & Negative word or Chinese & Visual valence words and & 64 \\
& accent & accent & auditory accented phrases & \\
B5 & Chinese accent & American accent & Auditory accented phrases & 32 \\
B6 & Positive word or Chinese & Negative word or American & Visual valence words and & 64 \\
& accent & accent & auditory accented phrases & \\
B7 & Positive word or Chinese & Negative word or American & Visual valence words and & 64 \\
& accent & accent & auditory accented phrases & \\
\hline
\end{tabular}

*Both the on screen category positions and the order of B2-4 and B5-7 were counterbalanced across participants.

For each block, the two characteristics were presented at the top left and right of the screen and participants were instructed to press the E key on the keyboard if the stimulus matched the characteristic on the right (e.g. "Positive") and press the I key if it matched the characteristic on the left (e.g. "Negative"). B1 was a practice block in which participants sort the valence words as positive or negative. The eight positive and eight negative visual stimuli were each repeated twice giving a total of 32 trials. B2 was another practice block, this time sorting the auditory stimuli as either Chinese-accented or American-accented. The eight Chinese stimuli and eight American stimuli were each presented twice, again giving 32 total trials for B2. B3 and B4 were combinations of blocks one and two in which participants sort both the visual and auditory stimuli into combined accent/valence categories. B3 and B4 were identical, but they were separated for analysis. All stimuli from B1 and B2 were used and each was repeated twice giving 64 total trials for each of B3 and B4. B5 was a repetition of B2 with the positions of the attribute categories switched on screen. B6 and B7 were repetitions of B3 and B4, now with the new attribute category positions from B5 and thus with reversed accent/valence associations. The 
order of trials within each block was randomized for each participant. Participants were instructed to complete trials as quickly as is possible without sacrificing accuracy.

\section{LEAP-Q and accent exposure measures.}

This study used a modified version of the LEAP-Q from Marian, Blumenfeld and Kaushanskaya (2003) to record participants' language backgrounds and to assess their levels of exposure to the foreign accent being tested. Because the targeted participants were primarily monolingual American English speakers, the LEAP-Q was presented in an abbreviated form. Following the shortened LEAP-Q, an additional section asked about personal interactions participants have with individuals with the Chinese accents, the frequency with which they hear such accents in their environments, and any travel they may have taken to either mainland China, Taiwan, or Hong Kong. These three questions are taken as measures of accent exposure.

speech evaluation instrument (SEI). To measure participants' explicit attitudes toward Chinese accents, this study employed the Speech Evaluation Instrument (SEI) first described in Zahn and Hopper (1985). In this instrument, participants listened to two recordings of a 102word text that was purportedly an answering machine message for a doctor's office. The contents of the two recordings were identical, and they differed only in the accent of the speaker. The same two speakers who recorded the auditory IAT stimuli recorded the SEI texts. After each recording, participants were asked to rate the speaker on 30 scales based on a variety of personal characteristics. Each scale asked for a rating from one to seven in which the extremes were labeled with two contrasting personal qualities. These included characteristics such as whether the speaker sounded educated or uneducated, whether they sounded likable or unlikeable, and whether they sounded enthusiastic or shy. Participants were given no information about the speakers and were instructed to focus their assessments on the quality of the speakers' voices alone.

\section{Analysis of Data}

The IAT responses were processed according to the recommended scoring algorithm in Greenwald, Nosek, and Banaji (2003). This involved elimination of all trials with response latencies exceeding 10 seconds and elimination of all participants in which more than $10 \%$ of trials had response latencies shorter than 300 milliseconds. Latencies for each incorrect trial were 
then replaced with the latency until correct response, as all trials provided negative feedback after incorrect responses and required participants to follow up with the correct response. For each participant, mean latencies were then calculated for each of the combined task blocks: B3, B4, B6, and B7. Two pooled standard deviations were then calculated: one for the combined SD of B3 and B6 and another for the combined SD of B4 and B7. Two differences were then calculated: mean latency of B3 - mean latency of B6 and mean latency of B4 - mean latency of B7. Finally, each difference was divided by the associated pooled standard deviation, and the two resulting quotients were averaged. This provides what Greenwald et al. (2003) call the D measure, a measure of each participant's implicit association about the target characteristic.

The SEIs for each participant were processed according to the original findings of Zahn and Hopper (1985). For each of the accented SEIs, the 30 items were grouped according to the three factors identified by Zahn and Hopper, which they name "superiority," "attractiveness," and "dynamism." Mean ratings were calculated for each of these factors, as well as an overall mean rating for each accent. Four differences were then calculated, subtracting the overall and factor means of the Chinese test from their associated American English means. These differences are taken as measures of the participant's explicit attitudes toward Chinese accented English relative to American English, both overall and with regard to the three specified factors.

Pearson's correlation coefficients were then calculated between the results of the two tests described above. Specifically, they tested whether participants' implicit attitudes about Chinese accents correlated with their overall explicit attitudes, or with any of the three identified subscales. Coefficients were also calculated for the correlation between IAT score and accent exposure.

\section{Results}

\section{IAT Scores}

When analyzing the IAT, possible scores range between 2 and -2. In this case, a positive score indicates a preference for American accents over Chinese accents, while a negative score indicates a preference for Chinese accents over American ones. Using the scoring ranges established by Nozeck, Bananji, and Greenwald (2005), an absolute score of less than 0.15 indicates a slight preference, a score between 0.15 and 0.35 indicates a moderate preference, and a score above 0.65 indicates a strong preference. Based on these criteria, the majority of 
participants (18) exhibited moderate preference for the American accent. Two participants showed strong preference for the American accent and three only showed slight preference for the American accent. Three participants reversed the trend, showing preference for the Chinese accent over the American one. Two of these were categorized as having a slight preference and one was categorized as having a moderate preference. The mean, range, standard deviation, and the results of a one-sample t-test performed on participants' IAT scores are listed in Table 3 below:

Table 3. Results of IAT measures

\begin{tabular}{ccccccc}
\hline Mean & Range & Standard Deviation & T-value & df & P-value & Confidence interval \\
\hline 1.04 & $\begin{array}{c}-0.30 \text { to } \\
1.24\end{array}$ & 0.32 & 5.36 & 25 & $<0.0001$ & $0.19,0.45$ \\
\hline
\end{tabular}

These results support the hypothesis that, overall, the population has a slight to moderate implicit bias against Chinese accents.

\section{SEI Scores}

Four one-sample T-tests were also conducted on the SEI results: one for the overall difference between the American and Chinese ratings and one for the rating differences in each of the three subscales identified by Zahn and Hopper (1985). The means, ranges, standard deviations, and results of the one-sample T-tests are displayed in Table 4 below:

Table 4. Results of SEI measures (difference between American and Chinese accents)

\begin{tabular}{|c|c|c|c|c|c|c|c|}
\hline Scale & Mean & Range & $\begin{array}{l}\text { Standard } \\
\text { Deviation }\end{array}$ & T-value & df & P-value & $\begin{array}{c}\text { Confidence } \\
\text { interval }\end{array}$ \\
\hline Overall Measure & 1.04 & -0.37 to 2.03 & 0.63 & 8.42 & 25 & $<0.0001$ & $0.79,1.30$ \\
\hline — Superiority subscale & 1.68 & -0.17 to 3.17 & 0.79 & 10.75 & 25 & $<0.0001$ & $1.35,2.00$ \\
\hline - Attractiveness subscale & 0.38 & -1.18 to 2.18 & 0.82 & 2.38 & 25 & $<0.05$ & $0.05,0.71$ \\
\hline —Dynamism subscale & 1.00 & -2.29 to 2.86 & 1.10 & 4.64 & 25 & $<0.0001$ & $0.55,1.45$ \\
\hline
\end{tabular}

These results indicate that, overall, the population has significant negative explicit attitudes toward Chinese accents as well as implicit ones. These negative attitudes appear to be 
concentrated most heavily in the Superiority and Dynamism factors, although significant negative attitudes are present in the Attractiveness factor as well.

\section{Correlation between IAT, SEI, and exposure}

Five Pearson's correlation coefficients were calculated examining the level of correlation between participants' IAT scores and their SEI scores overall, their scores on each of the SEI subscales, and their self-reported level of accent exposure. To measure accent exposure, participants were asked to estimate how many times in the past week they had interacted with a person with a Chinese accent, as well as how many times they had overheard someone speaking with a Chinese accent, and the sum was taken as an estimate of accent exposure. The R, $\mathrm{R}^{2}$, and $\mathrm{P}$-values for each of these correlations are shown in Table 5 below:

Table 5. Results of coefficient calculations

\begin{tabular}{lccc}
\hline Comparison & $\mathrm{R}$ & $\mathrm{R}^{2}$ & P-value \\
\hline IAT/SEI (Overall) & 0.031 & $<0.01$ & 0.88 \\
IAT/SEI (Superiority) & -0.016 & $<0.01$ & 0.94 \\
IAT/SEI (Attractiveness) & 0.094 & $<0.01$ & 0.65 \\
IAT/SEI (Dynamism) & -0.013 & $<0.01$ & 0.95 \\
IAT/Exposure & 0.37 & 0.14 & 0.03 \\
\hline
\end{tabular}

No meaningful correlations were found between IAT scores and either the SEI scores overall or any of the subscales. A weak correlation was found between IAT scores and accent exposure. This correlation, though, moved opposite from what was expected: more frequent exposure to Chinese accents appears to correlate with more negative implicit attitudes towards that accent.

\section{Discussion}

Individually, the implicit and explicit attitude findings support the hypotheses. Participants exhibited negative explicit and implicit attitudes toward Chinese accents. The correlation results, on the other hand, did not support the hypotheses. No correlation was found between implicit and explicit attitudes. It is not clear why such correlations were not found in this study. Pantos and Perkins (2012) found previously that implicit attitudes about accents were, 
in fact, partially predicted by explicit attitudes. One likely limitation of the current study is its sample size. We were only able to collect data from 26 participants, which may not have given the statistical power necessary to detect the relevant effects. Furthermore, the correlation between exposure and IAT score is reverse to what was expected. More frequent Chinese accent exposure here appears to be associated with greater bias against Chinese accents, albeit loosely. This may be due to a number of factors. It is possible that greater interaction with Chineseaccented individuals does, in fact, inspire greater bias about Chinese accents. It could also be the case that negative attitudes about Chinese accents cause individuals to notice and remember such accents more easily. This could artificially inflate self-reports of accent exposure among those with negative attitudes towards that accent. The fact that the correlation is relatively weak and the small sample size of this study also allow for the possibility that the correlation found is not genuine and is simply an artifact of this sample. Future research is needed to confirm this correlation and, if genuine, to determine its underlying causes.

These findings, along with the previous work on this topic, suggest that the IAT does provide a potential method for evaluating individual level linguistic attitudes, even when such attitudes are unconscious or socially undesirable. Further research is needed to map the uses and limitations of both auditory IATs generally and specifically those aimed at evaluating linguistic attitudes. If a foundation is provided for linguistic IATs, they can provide a valuable way to study and control for social attitudes about language. In particular, it will allow researchers to better explore the underlying causes of previously observed social influences on linguistic processing.

\section{References}

Campbell-Kibler, K. (2012). The Implicit Association Test and sociolinguistic meaning. Lingua, 122(7), 753-763. https://doi.org/10.1016/j.lingua.2012.01.002

Dunham, Y., Baron, A. S., \& Banaji, M. R. (2016). The development of implicit gender attitudes. Developmental Science, 19(5), 781-789. https://doi.org/10.1111/desc.12321

Egloff, B., \& Schmukle, S.C. (2002). Predictive Validity of an Implicit Association Test for Assessing Anxiety, Journal of Personality and Social Psychology, 83(6): 14411455, https://doi.org/10.1037/0022-3514.83.6.1441

Friese, M., Smith, C. T., Plischke, T., Bluemke, M, \& Nosek, B. A. (2012). Do Implicit Attitudes Predict Actual Voting Behavior Particularly for Undecided Voters?. PLOS ONE, 7(8), e44130. https://doi.org/10.1371/journal.pone.0044130 
Greenwald, A. G., McGhee, D. E., \& Schwartz, M. (1998). Measuring individual differences in implicit cognition: The Implicit Association Test. Journal of Personality and Social Psychology.

Greenwald, A. G., Nosek, B. A., \& Banaji, M. R. (2003). Understanding and using the Implicit Association Test: I. An improved scoring algorithm. Journal of Personality and Social Psychology, 85(2), 197-216. http://dx.doi.org/10.1037/0022-3514.85.2.197

Greenwald, A. G., Poehlman, T. A., Uhlmann, E. L., \& Banaji, M. J. (2009). Understanding and using the implicit association test: III. Meta-analysis of predictive validity. Journal of Personality and Social Psychology, 97, 17-41. https://doi.org/10.1037/a0015575.

Gregg, A. P., \& Klymowsky, J. (2013). The Implicit Association Test in market research: Potentials and pitfalls. Psychology \& Marketing, 30(7), 588-601. http://dx.doi.org/10.1002/mar.20630

Haider, A. H., Schneider, E. B., Sriram, N., Scott, V. K., Swoboda, S. M., Zogg, C. K., .. Cooper, L. A. (2015). Original scientific article: Unconscious Race and Class Biases among Registered Nurses: Vignette-Based Study Using Implicit Association Testing. Journal of the American College of Surgeons, 220, 1077-1086. https://doi.org/10.1016/j.jamcollsurg.2015.01.065

Ksiazkiewicz, A., \& Hedrick, J. (2013). An Introduction to Implicit Attitudes in Political Science Research. PS: Political Science \& Politics, 46(3), 525-531. https://doi.org/10.1017/S1049096513000632

Marian, V., Blumenfeld, H. K., \& Kaushanskaya, M. (2007). The Language Experience and Proficiency Questionnaire (LEAP-Q): Assessing Language Profiles in Bilinguals and Multilinguals. Journal of Speech, Language, and Hearing Research, 50(4), 940-967. https://doi.org/10.1044/10924388(2007/067).

Nosek, B. A., Greenwald, A. G., \& Banaji, M. R. (2005). Understanding and Using the Implicit Association Test: II. Method Variables and Construct Validity. Personality and Social Psychology Bulletin, 31(2), 166-180. https://doi.org/10.1177/0146167204271418

Pantos, A. J., \& Perkins, A. W. (2012). Measuring Implicit and Explicit Attitudes Toward Foreign Accented Speech. Journal of Language and Social Psychology, 32(1), 3-20.

Pirutinsky, S., Carp, S., \& Rosmarin, D. H. (2017). A Paradigm to Assess Implicit Attitudes towards God: The Positive/Negative God Associations Task. Journal Of Religion And Health, 56(1), 305-319. https://doi.org/10.1007/s10943-016-0303-y

Rosseel, L., Speelman, D., \& Geeraerts, D. (2018). Measuring language attitudes using the Personalized Implicit Association Test: A case study on regional varieties of Dutch in Belgium. Journal of Linguistic Geography, 6(1), 20-39. https://doi.org/10.1017/jlg.2018.3

Westgate, E. C., Riskind, R. G., \& Nosek, B. A. (2015). Implicit preferences for straight people over lesbian women and gay men weakened from 2006 to 2013. Collabra, 1. https://doi.org/10.1525/collabra.18

Zahn, C. J., \& Hopper, R. (1985). Measuring Language Attitudes: The Speech Evaluation Instrument. Journal of Language and Social Psychology, 4(2), 113-123. https://doi.org/10.1177/0261927X8500400203 